Korean J. Math. 21 (2013), No. 2, pp. 171-180

http://dx.doi.org/10.11568/kjm.2013.21.2.171

\title{
FUZZY SEMIGROUPS IN REDUCTIVE SEMIGROUPS
}

\author{
INHEUNG CHON
}

\begin{abstract}
We consider a fuzzy semigroup $S$ in a right (or left) reductive semigroup $X$ such that $S(k)=1$ for some $k \in X$ and find a faithful representation (or anti-representation) of $S$ by transformations of $S$. Also we show that a fuzzy semigroup $S$ in a weakly reductive semigroup $X$ such that $S(k)=1$ for some $k \in X$ is isomorphic to the semigroup consisting of all pairs of inner right and left translations of $S$ and that $S$ can be embedded into the semigroup consisting of all pairs of linked right and left translations of $S$ with the property that $S$ is an ideal of the semigroup.
\end{abstract}

\section{Introduction}

The concept of fuzzy sets was first introduced by Zadeh ([11]). Rosenfeld ([9]) used this concept to formulate the notion of fuzzy groups. Kuroki ([5], [6], [7], [8]) introduced fuzzy semigroups, fuzzy ideals, fuzzy bi-ideals, and fuzzy semiprime ideals in semigroups, and developed some properties of those semigroups and ideals. Subsequently Dos ([4]) studied fuzzy regular subsemigroups in regular semigroups, fuzzy inverse subsemigroups in inverse semigroups, and fuzzy multiplication semigroups in commutative semigroups. As a continuation of these studies, we consider fuzzy semigroups in a reductive semigroup and find some properties of those fuzzy semigroups in this note.

Received March 21, 2013. Revised May 15, 2013. Accepted May 20, 2013.

2010 Mathematics Subject Classification: 20N25.

Key words and phrases: fuzzy semigroup, reductive semigroup, weakly reductive semigroup, linked, translational hull.

This work was supported by a special research grant from Seoul Women's University (2013).

(c) The Kangwon-Kyungki Mathematical Society, 2013.

This is an Open Access article distributed under the terms of the Creative commons Attribution Non-Commercial License (http://creativecommons.org/licenses/by -nc/3.0/) which permits unrestricted non-commercial use, distribution and reproduction in any medium, provided the original work is properly cited. 
In section 2 we review some basic definitions and properties of fuzzy sets, fuzzy points, and fuzzy semigroups which will be used in next sections. In section 3 we consider a fuzzy semigroup $S$ in a right (or left) reductive semigroup $X$ such that $S(k)=1$ for some $k \in X$, find a faithful representation (or anti-representation) of $S$, and show that $S$ is isomorphic (or anti-isomorphic) to the semigroup of all inner left (or right) translations of $S$. In section 4 we show that a fuzzy semigroup $S$ in a weakly reductive semigroup $X$ such that $S(k)=1$ for some $k \in X$ is isomorphic to a semigroup $H\left(S_{0}\right)$ which consists of all pairs of inner right and left translations of $S$ and show that $S$ can be embedded into a semigroup $H(S)$ which consists of all pairs of linked right and left translations of $S$ with the properties that $S$ is an ideal of $H(S)$ and every left (or right) translation of $S$ is induced by some inner left (or right) translation of $H(S)$ iff each left (or right) translation of $S$ is linked with some right (or left) translation of $S$.

\section{Preliminaries}

In this section we review some basic definitions and properties of fuzzy sets, fuzzy points, and fuzzy semigroups which will be used in section 3 and section 4 .

Definition 2.1. A function $B$ from a set $X$ to the closed unit interval $[0,1]$ in $\mathbb{R}$ is called a fuzzy set in $X$. For every $x \in B, B(x)$ is called a membership grade of $x$ in $B$. The set $\{x \in X: B(x)>0\}$ is called the support of $B$.

Definition 2.2. A $t$-norm is a function $T:[0,1] \times[0,1] \rightarrow[0,1]$ satisfying, for each $p, q, r, s$ in $[0,1]$,

(1) $T(p, 0)=0, T(p, 1)=p=T(1, p)$,

(2) $T(p, q) \leq T(r, s)$ if $p \leq r$ and $q \leq s$,

(3) $T(p, q)=T(q, p)$,

(4) $T(p, T(q, r))=T(T(p, q), r)$.

Definition 2.3. A t-norm $T:[0,1] \times[0,1] \rightarrow[0,1]$ is continuous if $T$ is continuous with respect to the usual topologies.

It is well known $([1])$ that the function $T_{m}:[0,1] \times[0,1] \rightarrow[0,1]$ defined by $T_{m}(a, b)=\min (a, b)$, the function $T_{p}:[0,1] \times[0,1] \rightarrow[0,1]$ 
defined by $T_{p}(a, b)=a b$, and the function $T_{M}:[0,1] \times[0,1] \rightarrow[0,1]$ defined by $T_{M}(a, b)=\max (a+b-1,0)$ are continuous t-norms.

The following definition is due to Sessa ([10]).

Definition 2.4. Let $X$ be a set and let $U, V$ be two fuzzy sets in $X$. Then $U \circ V$ is defined by

$$
(U \circ V)(x)= \begin{cases}\sup _{a b=x} T(U(a), V(b)) & \text { if } a b=x \\ 0 & \text { if } a b \neq x .\end{cases}
$$

Definition 2.5. A fuzzy set in a set $X$ is called a fuzzy point iff it takes the value 0 for all $y \in X$ except one, say, $x \in X$. If its value at $x$ is $\alpha(0<\alpha \leq 1)$, we denote this fuzzy point by $x_{\alpha}$, where the point $x$ is called its support. The fuzzy point $x_{\alpha}$ is said to be contained in a fuzzy set $A$, denoted by $x_{\alpha} \in A$, iff $\alpha \leq A(x)$.

Proposition 2.6. Let $x_{p}, y_{q}$ be fuzzy points in a groupoid $X$. Then $x_{p} \circ y_{q}=(x y)_{T(p, q)}$.

Proof. If $z=x y$, then

$$
\begin{aligned}
\left(x_{p} \circ y_{q}\right)(z) & =\left(x_{p} \circ y_{q}\right)(x y)=\sup _{a b=x y} T\left(x_{p}(a), y_{q}(b)\right) \\
& =T\left(x_{p}(x), y_{q}(y)\right)=T(p, q)
\end{aligned}
$$

If $z \neq x y$,

$$
\left(x_{p} \circ y_{q}\right)(z)=\sup _{b=z} T\left(x_{p}(a), y_{q}(b)\right)=0 .
$$

Thus, $x_{p} \circ y_{q}=(x y)_{T(p, q)}$.

Proposition 2.7. Let $A, B, C$ be fuzzy sets in a set $X$ and let $T$ be a continuous t-norm. If $X$ is associative, then $(A \circ B) \circ C=A \circ(B \circ C)$.

Proof. See Proposition 2.8 of [2].

From now on, we assume that every t-norm in this paper is continuous.

The following definition is due to Anthony and Sherwood ([1]). That is, they replaced the minimum condition proposed by Rosenfeld ([9]) with a t-norm. 
Definition 2.8. Let $X$ be a groupoid and $T$ be a t-norm. A function $S: X \rightarrow[0,1]$ is a fuzzy groupoid in $X$ iff for every $x, y$ in $X, S(x y) \geq$ $T(S(x), S(y))$. If $X$ is a group, a fuzzy groupoid $G$ is a fuzzy group in $X$ iff for each $x \in X, G\left(x^{-1}\right)=G(x)$.

See [1] for examples of fuzzy groups.

Proposition 2.9. Let $A$ be a non-empty fuzzy set of a groupoid $X$. Then the followings are equivalent.

(1) $A$ is a fuzzy groupoid.

(2) For any $x_{p}, y_{q} \in A, x_{p} \circ y_{q} \in A$.

(3) $A \circ A \subseteq A$.

Proof. See Proposition 2.7 of [2].

If $B$ is a fuzzy groupoid in a semigroup $X,\left(x_{p} \circ y_{q}\right) \circ z_{r}=x_{p} \circ$ $\left(y_{q} \circ z_{r}\right)$ for every $x_{p}, y_{q}, z_{r} \in B$ from Proposition 2.7. We call $B$ a fuzzy semigroup in $X$.

Example of a fuzzy semigroup. Let $X$ be a set of all natural numbers which are greater than or equal to 2 , that is, $X=\{2,3,4, \ldots\}$, and let - be a multiplication. Then $(X, \cdot)$ is a semigroup. Let $S: X \rightarrow[0,1]$ be a function defined by $S(a)=\frac{a}{a+1}$. Then $S(p \cdot q)=\frac{p \cdot q}{p \cdot q+1}$, and hence $S(p \cdot q) \geq S(q)$. Thus

$$
S(p \cdot q)=T(1, S(p \cdot q)) \geq T(S(p), S(p \cdot q)) \geq T(S(p), S(q)) .
$$

That is, $S$ is a fuzzy semigroup in $S$.

We write $x_{p} y_{q}$ for $x_{p} \circ y_{q}$ in the next section 3 and section 4 .

\section{Representations of fuzzy semigroups in a reductive semi- group}

In this section we discuss the representations of fuzzy semigroups in a semigroup and a reductive semigroup. First of all, we define a left and a right translation of a fuzzy semigroup which play important roles for the representations of fuzzy semigroups in semigroups.

Definition 3.1. Let $S$ be a fuzzy semigroup in a semigroup $X$. A transformation $l: S \rightarrow S$ is called a left translation of $S$ if $l\left(x_{p}\right) y_{q}=$ $l\left(x_{p} y_{q}\right)$ for all $x_{p}, y_{q} \in S$. A transformation $r: S \rightarrow S$ is called a right translation of $S$ if $x_{p} r\left(y_{q}\right)=r\left(x_{p} y_{q}\right)$ for all $x_{p}, y_{q} \in S$. 
It is easily checked that a transformation $l_{a_{p}}: S \rightarrow S$ defined by $l_{a_{p}}\left(b_{q}\right)=a_{p} b_{q}$ for $a_{p} \in S$ is a left translation and a transformation $r_{a_{p}}: S \rightarrow S$ defind by $r_{a_{p}}\left(b_{q}\right)=b_{q} a_{p}$ for $a_{p} \in S$ is a right translation. We call $l_{a_{p}}$ a inner left translation of $S$ and call $r_{a_{p}}$ a inner right translation of $S$.

Definition 3.2. Let $f$ be a mapping from a set $X$ to a set $Y$. Let $A$ be a fuzzy set in $X$. Then the image of $A$, written $f(A)$, is the fuzzy set in $Y$ with membership function defined by

$$
f(A)(y)=\left\{\begin{array}{cl}
\sup _{z \in f^{-1}(y)} A(z), & \text { if } f^{-1}(y) \text { is nonempty } \\
0, & \text { otherwise }
\end{array}\right.
$$

for all $y \in Y$.

Proposition 3.3. Let $S$ be a fuzzy semigroup in a set $X$. Then the set $\mathcal{W}_{S}$ of all transformations of $S$ forms a semigroup under the operation of composition 0 .

Proof. Let $f, g, h \in \mathcal{W}_{S}$ and let $x_{p} \in S$. Then it is easy to see $f\left(x_{p}\right)=[f(x)]_{p}$ from Definition 3.2. Since $x_{p} \in S$ and $f \in \mathcal{W}_{S}, f\left(x_{p}\right) \in$ $S$, and hence $[f(x)]_{p} \in S$. Since $g \in \mathcal{W}_{S}, g\left([f(x)]_{p}\right) \in S$. Since $(g \circ$ $f)\left(x_{p}\right)=g\left([f(x)]_{p}\right),(g \circ f)\left(x_{p}\right) \in S$. That is, $g \circ f \in \mathcal{W}_{S}$. Clearly $[(f \circ g) \circ h]\left(x_{p}\right)=[f \circ(g \circ h)]\left(x_{p}\right)$.

We define a representation of a fuzzy semigroup in a semigroup and find a representation of the fuzzy semigroup.

Definition 3.4. Let $S$ be a fuzzy semigroup in a semigroup X, let $L$ be a fuzzy set, and let $\mathcal{W}_{L}$ be the semigroup of all transformations of $L$. A homomorphism $\psi: S \rightarrow \mathcal{W}_{L}$ is called a representation of $S$ by transformations of $L$ and a representation $\psi$ of $S$ is called faithful if it is one-to-one. An anti-homomorphism $\phi: S \rightarrow \mathcal{W}_{L}$ is called an antirepresentation of $S$ by transformations of $L$ and an anti-representation $\phi$ of $S$ is called faithful if it is one-to-one.

Proposition 3.5. Let $S$ be a fuzzy semigroup in a semigroup $X$ and let $\mathcal{W}_{S}$ be the semigroup of all transformations of $S$. Then there is a representation $\psi: S \rightarrow \mathcal{W}_{S}$ of $S$ and there is an anti-representation $\phi: S \rightarrow \mathcal{W}_{S}$ of $S$.

Proof. Let $r_{a_{p}}: S \rightarrow S$ be an inner right translation and let $l_{a_{p}}$ : $S \rightarrow S$ be an inner left translation. Let $\psi: S \rightarrow \mathcal{W}_{S}$ be a map defined 
by $\psi\left(a_{p}\right)=l_{a_{p}}$ and let $\phi: S \rightarrow \mathcal{W}_{S}$ be a map defined by $\phi\left(a_{p}\right)=r_{a_{p}}$. Then $\left[\psi\left(a_{p} b_{q}\right)\right]\left(c_{r}\right)=l_{a_{p} b_{q}}\left(c_{r}\right)=\left(a_{p} b_{q}\right) c_{r} . \quad\left[\psi\left(a_{p}\right) \circ \psi\left(b_{q}\right)\right]\left(c_{r}\right)=\left(l_{a_{p}} \circ\right.$ $\left.l_{b_{q}}\right)\left(c_{r}\right)=l_{a_{p}}\left(b_{q} c_{r}\right)=a_{p}\left(b_{q} c_{r}\right)$. Since $X$ is associative, $\left(a_{p} b_{q}\right) c_{r}=a_{p}\left(b_{q} c_{r}\right)$ by Proposition 2.7. Thus, $\psi\left(a_{p} b_{q}\right)=\psi\left(a_{p}\right) \circ \psi\left(b_{q}\right)$. Similarly we may show $\phi\left(a_{p} b_{q}\right)=\phi\left(b_{q}\right) \circ \phi\left(a_{p}\right)$.

We now turn to a faithful representation of a fuzzy semigroup in a reductive semigroup.

Definition 3.6. A semigroup $X$ is called right reductive if $a x=b x$ for all $x \in X$ implies $a=b$. A semigroup $X$ is called left reductive if $x a=x b$ for all $x \in X$ implies $a=b$. A semigroup $X$ is called reductive if $X$ is right reductive and left reductive. A semigroup $X$ is called weakly reductive if $a x=b x$ and $x a=x b$ for all $x \in X$ imply $a=b$.

TheOrem 3.7. Let $S$ be a fuzzy semigroup in a right (or left) reductive semigroup $X$ such that $S(k)=1$ for some $k \in X$ and let $\mathcal{W}_{S}$ be the semigroup of all transformations of $S$. Then there is a faithful representation $\psi: S \rightarrow \mathcal{W}_{S}$ of $S$ (or a faithful anti-representation $\phi: S \rightarrow \mathcal{W}_{S}$ of $S)$.

Proof. Let $l_{a_{p}}: S \rightarrow S$ be an inner left translation and let $\psi: S \rightarrow$ $\mathcal{W}_{S}$ be a map defined by $\psi\left(a_{p}\right)=l_{a_{p}}$. Then $\psi$ is a representation of $S$ by Proposition 3.5. Suppose $\psi\left(a_{p}\right)=\psi\left(b_{q}\right)$. Then $l_{a_{p}}=l_{b_{q}}$. For all $c_{r} \in S, l_{a_{p}}\left(c_{r}\right)=l_{b_{q}}\left(c_{r}\right)$, that is, $a_{p} c_{r}=b_{q} c_{r}$. By Proposition 2.6, $(a c)_{T(p, r)}=(b c)_{T(q, r)}$ for all $c_{r} \in S$. Since $a, b$, and $c$ are in a right reductive semigroup $X, a=b$. Since $S(k)=1, k_{1} \in S$, and hence $a_{p} k_{1}=$ $b_{q} k_{1}$. That is, $(a k)_{T(p, 1)}=(b k)_{T(q, 1)}$. Since $a=b, T(p, 1)=T(q, 1)$, and hence $p=q$. Thus, $a_{p}=b_{q}$, that is, $\psi$ is injective. Similarly we may show that for an inner right translation $r_{a_{p}}$ of $S$, a map $\phi: S \rightarrow \mathcal{W}_{S}$ defined by $\phi\left(a_{p}\right)=r_{a_{p}}$ is a faithful anti-representation.

Corollary 3.8. Let $S$ be a fuzzy semigroup in a reductive semigroup $X$ such that $S(k)=1$ for some $k \in X$ and let $\mathcal{W}_{S}$ be the semigroup of all transformations of $S$. Then there are a faithful representation $\psi: S \rightarrow \mathcal{W}_{S}$ of $S$ and a faithful anti-representation $\phi: S \rightarrow \mathcal{W}_{S}$ of $S$.

Proof. Immediate from Theorem 3.7.

Theorem 3.9. Let $S$ be a fuzzy semigroup in a right (or left) reductive semigroup $X$ such that $S(k)=1$ for some $k \in X$. Then $S$ is isomorphic (or anti-isomorphic) to the semigroup of all inner left (or right) translations of $S$. 
Proof. Let $\mathcal{W}_{S}^{*}$ be the set of all inner left translations of $S$. Let $l_{a_{p}}, l_{b_{q}} \in \mathcal{W}_{S}^{*}$. Then $\left(l_{a_{p}} \circ l_{b_{q}}\right)\left(c_{r}\right)=a_{p}\left(b_{q} c_{r}\right)$ and $l_{a_{p} b_{q}}\left(c_{r}\right)=\left(a_{p} b_{q}\right) c_{r}$. Since $S$ is a fuzzy semigroup, $a_{p} b_{q} \in S$ and $\left(a_{p} b_{q}\right) c_{r} \in S$ from Proposition 2.9. Thus, $l_{a_{p} b_{q}} \in W_{S}^{*}$. Since $X$ is associative, $a_{p}\left(b_{q} c_{r}\right)=\left(a_{p} b_{q}\right) c_{r}$ from Proposition 2.7, and hence, $l_{a_{p} b_{q}}=l_{a_{p}} \circ l_{b_{q}}$. Thus $l_{a_{p}} \circ l_{b_{q}} \in W_{S}^{*}$. Clearly $\left[\left(l_{a_{p}} \circ l_{b_{q}}\right) \circ l_{c_{r}}\right]\left(x_{t}\right)=\left[l_{a_{p}} \circ\left(l_{b_{q}} \circ l_{c_{r}}\right)\right]\left(x_{t}\right)$. Hence, $W_{S}^{*}$ is a semigroup. Let $\psi: S \rightarrow \mathcal{W}_{S}^{*}$ be a map defined by $\psi\left(a_{p}\right)=l_{a_{p}}$. Then $\psi$ is an isomorphism by Theorem 3.7. Similarly we may prove that $S$ is anti-isomorphic to the semigroup of all inner right translations of $S$.

Corollary 3.10. Let $S$ be a fuzzy semigroup in a reductive semigroup $X$ such that $S(k)=1$ for some $k \in X$. Then $S$ is isomorphic to the semigroup of all inner left translations of $S$ and $S$ is anti-isomorphic to the semigroup of of all inner right translations of $S$.

Proof. Immediate from Theorem 3.9.

\section{Embedding of fuzzy semigroups into semigroups}

In this section we discuss the embedding problem of a fuzzy semigroup in a reductive semigroup into a semigroup. First we define a translational hull of a fuzzy semigroup into which the fuzzy semigroup is embedded.

Definition 4.1. A right translation $r$ and a left translation $l$ of a fuzzy semigroup $S$ in a semigroup $X$ are said to be linked if $x_{p} l\left(y_{q}\right)=$ $r\left(x_{p}\right) y_{q}$. The set of all pairs $(r, l)$ of linked right and left translations $r$ and $l$ of $S$ is called the translational hull of $S$ and is denoted by $H(S)$.

We define an operation in $H(S)$ by $(r, l)\left(r^{\prime}, l^{\prime}\right)=\left(r^{\prime} \circ r, l \circ l^{\prime}\right)$ for $(r, l),\left(r^{\prime}, l^{\prime}\right) \in H(S)$. The following proposition shows that $H(S)$ is a semigroup under this operation.

Proposition 4.2. The translational hull $H(S)$ of a fuzzy semigroup $S$ in a semigroup $X$ is a semigroup.

Proof. Let $(r, l),\left(r^{\prime}, l^{\prime}\right) \in H(S)$. It is easy to check that $r^{\prime} \circ r$ is a right translation of $S$ and $l \circ l^{\prime}$ is a left translation of $S$. Since $(r, l)$ and $\left(r^{\prime}, l^{\prime}\right)$ are linked pairs, $a_{p} l\left(b_{q}\right)=r\left(a_{p}\right) b_{q}$ and $a_{p} l^{\prime}\left(b_{q}\right)=r^{\prime}\left(a_{p}\right) b_{q}$ for all $a_{p}, b_{q} \in S . a_{p}\left[\left(l \circ l^{\prime}\right)\left(b_{q}\right)\right]=a_{p}\left[l\left(l^{\prime}\left(b_{q}\right)\right)\right]=r\left(a_{p}\right) l^{\prime}\left(b_{q}\right)=r^{\prime}\left(r\left(a_{p}\right)\right) b_{q}=\left[\left(r^{\prime} \circ\right.\right.$ $\left.r)\left(a_{p}\right)\right] b_{q}$. Thus, $a_{p}\left[\left(l \circ l^{\prime}\right)\left(b_{q}\right)\right]=\left[\left(r^{\prime} \circ r\right)\left(a_{p}\right)\right] b_{q}$, that is, $(r, l)\left(r^{\prime}, l^{\prime}\right)=\left(r^{\prime} \circ\right.$ 
$\left.r, l \circ l^{\prime}\right) \in H(S)$. Let $\left(r_{1}, l_{1}\right),\left(r_{2}, l_{2}\right),\left(r_{3}, l_{3}\right) \in H(S)$. $\left[\left(r_{1}, l_{1}\right)\left(r_{2}, l_{2}\right)\right]\left(r_{3}, l_{3}\right)=$ $\left(r_{2} \circ r_{1}, l_{1} \circ l_{2}\right)\left(r_{3}, l_{3}\right)=\left(r_{3} \circ r_{2} \circ r_{1}, l_{1} \circ l_{2} \circ l_{3}\right) .\left(r_{1}, l_{1}\right)\left[\left(r_{2}, l_{2}\right)\left(r_{3}, l_{3}\right)\right]=$ $\left(r_{1}, l_{1}\right)\left(r_{3} \circ r_{2}, l_{2} \circ l_{3}\right)=\left(r_{3} \circ r_{2} \circ r_{1}, l_{1} \circ l_{2} \circ l_{3}\right)$. Thus, $H(S)$ is associative.

For a fuzzy semigroup $S$ in a semigroup $X$, let $H\left(S_{0}\right)=\left\{\left(r_{a_{p}}, l_{a_{p}}\right)\right.$ : $\left.a_{p} \in S\right\}$, where $r_{a_{p}}$ is an inner right translation of $S$ and $l_{a_{p}}$ is an inner left translation of $S$. It is esay to see that $r_{a_{p}}$ and $l_{a_{p}}$ are linked, that is, $H\left(S_{0}\right) \subset H(S)$. We characterize $H\left(S_{0}\right)$ in Theorem 4.3 and Lemma 4.5.

THEOREM 4.3. Let $S$ be a fuzzy semigroup in a weakly reductive semigroup $X$ such that $S(k)=1$ for some $k \in X$. Then $H\left(S_{0}\right)$ is a subsemigroup of $H(S)$ and $S$ is isomorphic to $H\left(S_{0}\right)$.

Proof. Let $\left(r_{a_{p}}, l_{a_{p}}\right),\left(r_{b_{q}}, l_{b_{q}}\right) \in H\left(S_{0}\right)$. Then $\left(r_{b_{q}} \circ r_{a_{p}}\right)\left(c_{r}\right)=\left(c_{r} a_{p}\right) b_{q}$ $=r_{a_{p} b_{q}}\left(c_{r}\right)$ and $\left(l_{a_{p}} \circ l_{b_{q}}\right)\left(c_{r}\right)=a_{p}\left(b_{q} c_{r}\right)=l_{a_{p} b_{q}}\left(c_{r}\right)$. Thus, $\left(r_{a_{p}}, l_{a_{p}}\right)\left(r_{b_{q}}, l_{b_{q}}\right)$ $=\left(r_{b_{q}} \circ r_{a_{p}}, l_{a_{p}} \circ l_{b_{q}}\right)=\left(r_{a_{p} b_{q}}, l_{a_{p} b_{q}}\right)$. Since $a_{p}, b_{q} \in S, a_{p} b_{q} \in S$ from Proposition 2.9. Hence, $\left(r_{a_{p}}, l_{a_{p}}\right)\left(r_{b_{q}}, l_{b_{q}}\right) \in H\left(S_{0}\right)$. Clearly $H\left(S_{0}\right)$ is associative. Thus, $H\left(S_{0}\right)$ is a subsemigroup of $H(S)$. Let $\psi: S \rightarrow H\left(S_{0}\right)$ be a map defined by $\psi\left(a_{p}\right)=\left(r_{a_{p}}, l_{a_{p}}\right)$. Then $\psi\left(a_{p} b_{q}\right)=\left(r_{a_{p} b_{q}}, l_{a_{p} b_{q}}\right)=$ $\left(r_{b_{q}} \circ r_{a_{p}}, l_{a_{p}} \circ l_{b_{q}}\right)=\left(r_{a_{p}}, l_{a_{p}}\right)\left(r_{b_{q}}, l_{b_{q}}\right)=\psi\left(a_{p}\right) \psi\left(b_{q}\right)$. Suppose $\psi\left(a_{p}\right)=$ $\psi\left(b_{q}\right)$. Then $\left(r_{a_{p}}, l_{a_{p}}\right)=\left(r_{b_{q}}, l_{b_{q}}\right)$, that is, $r_{a_{p}}=r_{b_{q}}$ and $l_{a_{p}}=l_{b_{q}}$. Since $r_{a_{p}}\left(c_{r}\right)=r_{b_{q}}\left(c_{r}\right)$ for all $c_{r} \in S, c_{r} a_{p}=c_{r} b_{q}$. By Proposition 2.6, $(c a)_{T(r, p)}=(c b)_{T(r, q)}$ for all $c_{r} \in S$. Thus, $c a=c b$ for all $c \in X$. Since $l_{a_{p}}\left(c_{r}\right)=l_{b_{q}}\left(c_{r}\right)$ for all $c_{r} \in S, a_{p} c_{r}=b_{q} c_{r}$. By Proposition 2.6, $(a c)_{T(p, r)}=(b c)_{T(q, r)}$ for all $c_{r} \in S$. Thus $a c=b c$ for all $c \in X$. Since $a, b, c$ are in a weakly reductive semigroup $X, a=b$. Since $S(k)=1$, $k_{1} \in S$, and hence, $(k a)_{T(1, p)}=(k b)_{T(1, q)}$. Since $a=b, T(1, p)=T(1, q)$, and hence, $p=q$. Thus, $a_{p}=b_{q}$, that is, $\psi$ is injective. Clearly $\psi$ is surjective. Hence, $S$ is isomorphic to $H\left(S_{0}\right)$.

Definition 4.4. A non-empty set $L$ (or $R$ ) of a semigroup $S$ is a left ideal (or right ideal) of $S$ if $S L \subseteq L$ (or $R S \subseteq R$ ). I is an ideal of $S$ if $I S \cup S I \subseteq I$.

Lemma 4.5. For a fuzzy semigroup $S$ in a semigroup $X, H\left(S_{0}\right)$ is an ideal of $H(S)$.

Proof. Let $g \in H\left(S_{0}\right) H(S)$. Then $g=\left(r_{a_{p}}, l_{a_{p}}\right)(r, l)=\left(r \circ r_{a_{p}}, l_{a_{p}} \circ\right.$ $l)$, where $r$ and $l$ are linked. Since $r_{r\left(a_{p}\right)}\left(b_{q}\right)=b_{q} r\left(a_{p}\right)=r\left(b_{q} a_{p}\right)=$ $r\left(r_{a_{p}}\left(b_{q}\right)\right)=\left(r \circ r_{a_{p}}\right)\left(b_{q}\right), r_{r\left(a_{p}\right)}=r \circ r_{a_{p}}$. Since $a_{p} l\left(b_{q}\right)=r\left(a_{p}\right) b_{q},\left(l_{a_{p}} \circ\right.$ $l)\left(b_{q}\right)=l_{a_{p}}\left(l\left(b_{q}\right)\right)=a_{p} l\left(b_{q}\right)=r\left(a_{p}\right) b_{q}=l_{r\left(a_{p}\right)} b_{q}$, that is, $\left(l_{a_{p}} \circ l\right)=$ 
$l_{r\left(a_{p}\right)}$. Thus, $g=\left(r \circ r_{a_{p}}, l_{a_{p}} \circ l\right)=\left(r_{r\left(a_{p}\right)}, l_{r\left(a_{p}\right)}\right) \in H\left(S_{0}\right)$. That is, $H\left(S_{0}\right) H(S) \subseteq H\left(S_{0}\right)$. Let $g \in H(S) H\left(S_{0}\right)$. Then $g=(r, l)\left(r_{a_{p}}, l_{a_{p}}\right)=$ $\left(r_{a_{p}} \circ r, l \circ l_{a_{p}}\right)$, where $r$ and $l$ are linked. Since $l_{l\left(a_{p}\right)}\left(b_{q}\right)=l\left(a_{p}\right) b_{q}=$ $l\left(a_{p} b_{q}\right)=l\left(l_{a_{p}}\left(b_{q}\right)\right)=\left(l \circ l_{a_{p}}\right)\left(b_{q}\right), l_{l\left(a_{p}\right)}=l \circ l_{a_{p}}$. Since $b_{q} l\left(a_{p}\right)=r\left(b_{q}\right) a_{p}$, $\left(r_{a_{p}} \circ r\right)\left(b_{q}\right)=r_{a_{p}}\left(r\left(b_{q}\right)\right)=r\left(b_{q}\right) a_{p}=b_{q} l\left(a_{p}\right)=r_{l\left(a_{p}\right)}\left(b_{q}\right)$, that is, $r_{a_{p}} \circ r=$ $r_{l\left(a_{p}\right)}$. Thus, $g=\left(r_{a_{p}} \circ r, l \circ l_{a_{p}}\right)=\left(r_{l\left(a_{p}\right)}, l_{l\left(a_{p}\right)}\right) \in H\left(S_{0}\right)$. That is, $H(S) H\left(S_{0}\right) \subseteq H\left(S_{0}\right)$. Hence, $H\left(S_{0}\right)$ is an ideal of $H(S)$.

It is well known ([3]) that a weakly reductive semigroup $S$ can be embedded in a semigroup $T$ with the properties that $S$ is an ideal of $T$ and every left (or right) translation of $S$ is induced by some inner left (or right) translation of $T$ iff each left (or right) translation of $S$ is linked with some right (or left) translation of $S$. The following theorem may be considered as the corresponding one in fuzzy semigroups.

THEOREM 4.6. Let $S$ be a fuzzy semigroup on a weakly reductive semigroup $X$ such that $S(k)=1$ for some $k \in X$. Then $S$ can be embedded into a semigroup $H(S)$ with the properties that

(1) $S$ is an ideal of $H(S)$.

(2) every left (or right) translation of $S$ is induced by some inner left (or right) translation of $H(S)$ iff each left (or right) translation of $S$ is linked with some right (or left) translation of $S$.

Proof. We may identify $S$ with $H\left(S_{0}\right)$ by Theorem 4.3. By Lemma 4.5, $S$ is an ideal of $H(S)$.

Suppose $l$ is a left translation of $S$ such that $(r, l) \in H(S)$ for some right translation $r$ of $S$. Since $r_{a_{p}} \circ r=r_{l\left(a_{p}\right)}$ and $l \circ l_{a_{p}}=l_{l\left(a_{p}\right)}$, $(r, l)\left(r_{a_{p}}, l_{a_{p}}\right)=\left(r_{a_{p}} \circ r, l \circ l_{a_{p}}\right)=\left(r_{l\left(a_{p}\right)}, l_{l\left(a_{p}\right)}\right)$. We may identify $a_{p}$ with $\left(r_{a_{p}}, l_{a_{p}}\right)$ and identify $l\left(a_{p}\right)$ with $\left(r_{l\left(a_{p}\right)}, l_{l\left(a_{p}\right)}\right)$ from Theorem 4.3. By the identification, $(r, l)\left(a_{p}\right)=(r, l)\left(r_{a_{p}}, l_{a_{p}}\right)=\left(r_{l\left(a_{p}\right)}, l_{l\left(a_{p}\right)}\right)=l\left(a_{p}\right)$. Thus, $l_{(r, l)}\left(a_{p}\right)=(r, l) a_{p}=l\left(a_{p}\right)$. Hence, $l=\left.l_{(r, l)}\right|_{S}$. Conversely, suppose that the left translation $l$ is induced by some inner left translation of $H(S)$, that is, $l=\left.l_{\left(r_{1}, l_{1}\right)}\right|_{S}$ for some right translation $r_{1}$ of $S$ and some left translation $l_{1}$ of $S$. Then $x_{p} l\left(y_{q}\right)=x_{p} l_{\left(r_{1}, l_{1}\right)}\left(y_{q}\right)=x_{p}\left(r_{1}, l_{1}\right)\left(y_{q}\right)$. Let $r=\left.r_{\left(r_{1}, l_{1}\right)}\right|_{S}$. Then $r$ is a right translation of $S$ and $r\left(x_{p}\right) y_{q}=$ $r_{\left(r_{1}, l_{1}\right)}\left(x_{p}\right) y_{q}=x_{p}\left(r_{1}, l_{1}\right)\left(y_{q}\right)$. Thus, $x_{p} l\left(y_{q}\right)=r\left(x_{p}\right) y_{q}$, that is, $r$ and $l$ are linked. Similarly we may prove the dual case. 


\section{References}

[1] J.M. Anthony and H. Sherwood, Fuzzy groups redefined, J. Math. Anal. Appl. 69 (1979), 124-130.

[2] I. Chon, Fuzzy ideals generated by fuzzy subsets in semigroups, (To appear Commun. Korean Math. Soc.).

[3] A.H. Clifford and G.B. Preston, The algebraic theory of semigroups Vol. 1, American mathematical society, Providence, Rhode Island, 1961.

[4] P. Da, Fuzzy multiplication semigroup, Fuzzy Sets and Systems 105 (1999), 171-176.

[5] N. Kuroki, On fuzzy ideals and fuzzy bi-ideals in semigroups, Fuzzy Sets and Systems 5 (1981), 203-215.

[6] N. Kuroki, Fuzzy semiprime ideals in semigroups, Fuzzy Sets and Systems 8 (1982), 71-79.

[7] N. Kuroki, On Fuzzy semigroups, Information Sciences 53 (1991), 203-236.

[8] N. Kuroki, Fuzzy semiprime quasi-ideals in semigroups, Information Sciences $\mathbf{7 5}$ (1993), 201-211.

[9] A. Rosenfeld, Fuzzy Groups, J. Math. Anal. Appl. 35 (1971), 512-517.

[10] S. Sessa, On fuzzy subgroups and fuzzy ideals under triangular norms, Fuzzy Sets and Systems 13 (1984), 95-100.

[11] L.A. Zadeh, Fuzzy sets, Inform. and Control 8 (1965), 338-353.

Department of Mathematics

Seoul Women's University

Seoul 139-774, Korea

E-mail: ihchon@swu.ac.kr 